\title{
Non-invasive investigation of kidney disease in type 1 diabetes by magnetic resonance imaging
}

\author{
P. E. Thelwall $\cdot$ R. Taylor $\cdot$ S. M. Marshall
}

Received: 6 January 2011 / Accepted: 23 March 2011 / Published online: 1 May 2011

(C) Springer-Verlag 2011

\begin{abstract}
Aims/hypothesis Pathophysiological abnormalities in early diabetic nephropathy are poorly understood. We employed MRI to characterise renal perfusion, tissue oxygenation and kidney size in non-diabetic volunteers and type 1 diabetic patients without and with early renal disease.

Methods We studied ten control participants (C; age 40.0 [range 31-54] years), nine longstanding normotensive type 1 diabetic patients (T1Normo; age 40.1 [31-50] years, estimated glomerular filtration rate [eGFR] $83.4 \pm$ $10.6 \mathrm{ml} \mathrm{min}^{-1} 1.73 \mathrm{~m}^{-2}$ ) and eight microalbuminuric type 1 diabetic patients (T1Micro; age 42.4 [33-52] years, eGFR $71.6 \pm 13.7 \mathrm{ml} \mathrm{min}^{-1} 1.73 \mathrm{~m}^{-2}$ ). Six microalbuminuric patients were restudied after 4 weeks without reninangiotensin-aldosterone system inhibitors. Phase contrast angiography and kidney blood oxygen level dependent (BOLD) $\left(R_{2}{ }^{*}\right)$ MRI were performed, before and during water diuresis. Contrast-enhanced MRI was performed at baseline urine flow rate. Renal artery flow, renal vascular resistance (RVR), cortical and medullary volumes, and $R_{2}{ }^{*}$ were determined.
\end{abstract}

Electronic supplementary material The online version of this article (doi:10.1007/s00125-011-2163-z) contains supplementary material, which is available to authorised users.

P. E. Thelwall $\cdot$ R. Taylor

Newcastle Magnetic Resonance Centre,

Campus for Ageing and Vitality, Newcastle University,

Newcastle upon Tyne NE4 5PL, UK

P. E. Thelwall $(\bowtie) \cdot$ R. Taylor $\cdot$ S. M. Marshall

Institute of Cellular Medicine, Newcastle University,

Newcastle upon Tyne, UK

e-mail: pete.thelwall@ncl.ac.uk
Results Renal cortical and medullary volumes were similar in all groups (cortex: C 108 \pm 16 , T1Normo 112 \pm 21 , T1Micro $111 \pm 10 \mathrm{~cm}^{3} / 1.73 \mathrm{~m}^{2}$; medulla: C $35 \pm 14$, T1Normo $29 \pm 10,33 \pm 6 \mathrm{~cm}^{3} / 1.73 \mathrm{~m}^{2}$ ). RVR increased from control to normoalbuminuric to microalbuminuric type 1 diabetic patients $(\mathrm{C} 0.061 \pm 0.018$, T1Normo $0.077 \pm$ 0.014, T1Micro $0.093 \pm 0.024 \mathrm{mmHg} \mathrm{m} l^{-1} \mathrm{~min}^{-1} 1.73 \mathrm{~m}^{-2}$, ANOVA $p=0.012$ ). RVR correlated inversely with eGFR in normoalbuminuric, but not in microalbuminuric diabetic patients. Renal artery flow was lower in the whole diabetes cohort (control $740 \pm 205$ vs diabetes $591 \pm 128 \mathrm{ml} \mathrm{min}^{-1}$ $\left.1.73 \mathrm{~m}^{-2}, p=0.035\right)$.

Conclusions/interpretation Cortical and medullary volumes remain normal in early diabetic nephropathy. Decreased renal flow in longstanding normoalbuminuric type 1 diabetic patients may reflect intrarenal vascular stiffening, whereas in the microalbuminuric patients it may also reflect increased intraglomerular pressure.

Keywords BOLD · Diabetic nephropathy $\cdot$ Magnetic resonance imaging $\cdot$ Microalbuminuria $\cdot$ Renal artery flow Renal vascular resistance $\cdot$ Type 1 diabetes mellitus

$\begin{array}{ll}\text { Abbreviations } \\ \text { 3D } & \text { Three-dimensional } \\ \text { ACR } & \text { Albumin:creatinine ratio } \\ \text { BOLD } & \text { Blood oxygen level dependent } \\ \text { C } & \text { Control participants } \\ \text { eGFR } & \text { Estimated glomerular filtration rate } \\ \text { NSF } & \text { Nephrogenic systemic fibrosis } \\ \text { RAAS } & \text { Renin-angiotensin-aldosterone system } \\ \text { ROI } & \text { Region of interest } \\ \text { RVR } & \text { Renal vascular resistance } \\ \text { T1Micro } & \text { Microalbuminuric type 1 diabetic patients }\end{array}$


T1Normo Longstanding, normoalbuminuric, normotensive type 1 diabetic patients

TE Echo time

TR Repetition time

NSA Number of signal averages

\section{Introduction}

Chronic kidney disease is common and presents an enormous burden to the individual and society. In 2008 in the UK, 6,639 patients began renal replacement therapy, with diabetes mellitus being the single commonest cause (24\%) [1]. Diabetic nephropathy results from a complex interaction between haemodynamic and metabolic factors [2]. Haemodynamic abnormalities arise from increased systemic, intrarenal and intraglomerular pressures, coupled with activation of the vasoactive hormone pathways favouring vasoconstriction. These haemodynamic changes activate intracellular signalling pathways and various prosclerotic, pro-inflammatory and permeability factors. Intracellular glucose-dependent pathways are also activated, resulting in increased oxidative stress and tissue hypoxia. The combination of all these factors leads eventually to increased permeability of the glomerulus and extracellular matrix accumulation, with eventual gradually increasing albuminuria, glomerulosclerosis and interstitial fibrosis resulting in end stage renal disease.

Unlike in other chronic kidney diseases, kidney size remains normal or even increased in diabetic nephropathy [3]. Expansion of both cortex and medulla occurs in animal models of diabetes [4], but there are no such data in type 1 diabetes, and it is not known whether the ratio of cortex to medulla alters as the disease progresses.

In short-duration type 1 diabetes, hyperglycaemia is associated with reduced renal vascular resistance (RVR) and hyperfiltration [5-7]. RVR increases with age in type 1 diabetic patients without complications, to a much greater extent than in non-diabetic participants [8], but it is not known whether there is a further increase in type 1 diabetic patients with early nephropathy, independent of age.

The complex pathophysiological changes underlying diabetic nephropathy ultimately lead to chronic tissue hypoxia [9]. The outer medulla is particularly sensitive to hypoxia because the active reabsorption process within the thick ascending loop of Henle requires a high level of oxygen consumption [10]. However, whether hypoxia is present in the early stages of nephropathy is not known.

MRI background Until recently, methods of investigating the pathophysiology of kidney disease have been limited, requiring radioisotopes, exposure to ionising radiation or renal biopsy. MRI is a powerful, flexible technique for measuring physiological function and imaging, which is safe and repeatable. Studies to date have shown that structural information about the renal parenchyma and vessels, and functional data such as perfusion, filtration and oxygenation can be obtained [11, 12]. Renal $R_{2}{ }^{*}$ measurements, typically referred to as BOLD (blood oxygen level dependent) contrast, have been developed to assess possible tissue hypoxia. These methods have been translated from brain imaging studies that employ changes in tissue $R_{2}{ }^{*}$ to report on neural activity via blood oxygenation [13]. Applied to renal tissue, studies have suggested that changes in cortical and medullary oxygenation can be monitored via $R_{2}{ }^{*}$ mapping, and that tissue oxygenation change in response to physiological perturbations (including water loading) can differ between normal and disease states [14-19], including type 2 diabetes [20, 21].

This study was designed to determine whether there are abnormalities in cortical and medullary volumes, in renal blood flow and in medullary oxygenation in normoalbuminuric and microalbuminuric diabetic volunteers. Magnetic resonance (MR) techniques were employed, with and without water diuresis to stimulate medullary energy consumption.

\section{Methods}

The study was approved by the Newcastle and North Tyneside Local Research Ethics Committee 1 and all participants gave written informed consent.

Study volunteers The three study volunteer groups were of similar age to obviate age-related changes in RVR [8]. Type 1 diabetic volunteers were recruited from the Newcastle Diabetes Centre. Control volunteers (five men, five women; age 40.0 7.1 [range 31-54] years) were healthy non-diabetic individuals on no medication with blood pressure $<140 / 80 \mathrm{mmHg}$, recruited from the staff of Newcastle University.

Normoalbuminuric type 1 diabetic volunteers $(n=9)$ were selected to be at very low risk of diabetic nephropathy (type 1 diabetes duration $>20$ years, early morning urine albumin:creatinine ratio $<3.0 \mathrm{mg} / \mathrm{mmol}$ in all recorded measurements, no or minimal background retinopathy, blood pressure $<140 / 80 \mathrm{mmHg}$, with no antihypertensive therapy and no known other renal disease). Microalbuminuric volunteers were selected on the basis of exhibiting persistent microalbuminuria (early morning urine albumin: creatinine ratio $>5.0 \mathrm{mg} / \mathrm{mmol}$ in all measurements over the last 5 years, with significant diabetic retinopathy). The 
characteristics of the diabetic volunteers are shown in Table 1. Diabetic volunteers were recruited only if their estimated glomerular filtration rate (eGFR) was greater than $60 \mathrm{ml} \mathrm{min}{ }^{-1} 1.73 \mathrm{~m}^{-2}$ (CKD EPI equation [22]), to minimise the risk of nephrogenic systemic fibrosis (NSF) due to administration of gadolinium-based contrast agents. All microalbuminuric participants were prescribed at least one inhibitor of the renin-angiotensin-aldosterone system (RAAS), with six also taking additional antihypertensive therapy. Despite this, systolic and diastolic blood pressures were significantly higher in the microalbuminuric patients. Serum creatinine was also significantly higher, although the difference in eGFR was not statistically significant. Six randomly selected microalbuminuric volunteers were studied on two occasions, once on their usual antihypertensive medications, including RAAS inhibitors, and once 4 weeks after withdrawal of the RAAS inhibitor. During these 4 weeks, volunteers' blood pressure was measured weekly. Three patients required additional, non-RAAS inhibition therapy to maintain blood pressure control. There was no significant difference in systolic or diastolic blood pressure, serum creatinine, eGFR or ACR on or off the RAAS inhibitor (Table 1).

Experimental protocol Volunteers attended the Newcastle Magnetic Resonance Centre at 08.00 hours after fasting from 22:00 hours the previous evening. Intravenous cannulae were placed for administration of contrast agent, and for blood sampling and insulin infusion in diabetic volunteers. Blood glucose concentration was maintained between 4.0 and $6.0 \mathrm{mmol} / 1$ in diabetic volunteers for the $\sim 5 \mathrm{~h}$ duration of the study by infusion of insulin (Actrapid, NovoNordisk, Bagsværd, Denmark), to avoid acute effects of hyperglycaemia on renal haemodynamics [23]. Venous blood glucose concentration was assayed using a YSI 2300 STAT Plus (YSI, Yellow Springs, OH, USA). The MRI protocol commenced when blood glucose concentration had been stable for $30 \mathrm{~min}$. Blood glucose values were similar during all three scans in the diabetic groups, with no significant differences between the groups. Blood sampling and insulin infusion was not performed on control participants.

Water loading MRI scans to assess renal haemodynamics and oxygenation were acquired at baseline (after stabilisation of blood glucose concentration, if applicable) and following water loading. Volunteers were instructed to void before and after completion of the baseline MRI scans, to measure baseline urine production rate. Volunteers then consumed $20 \mathrm{ml}$ water/ $\mathrm{kg}$ body weight over a $15 \mathrm{~min}$ period, then urine samples were obtained every $15 \mathrm{~min}$. MRI scans were repeated when urine production exceeded $8 \mathrm{ml} / \mathrm{min}$. A final scan was performed for contrast agent- enhanced MRI when urine production rate had returned to baseline.

MRI All scans were acquired on a 3T Achieva scanner (Philips Medical Systems, Best, the Netherlands) using its body coil for RF transmission and a six-element cardiac receive coil. Three scan sessions were performed per experiment, the first two to measure renal haemodynamics via phase contrast angiography and $R_{2}{ }^{*}$ (BOLD) imaging, before and after water loading, and the third to assess kidney structure via contrast-enhanced three-dimensional (3D) gradient echo imaging.

Phase contrast angiography was performed during breath holding with a cardiac-triggered gradient echo sequence (repetition time $[\mathrm{TR}]=5.1 \mathrm{~ms}$, echo time $[\mathrm{TE}]=3.1 \mathrm{~ms}$, tip $=10^{\circ}$, one $6 \mathrm{~mm}$ slice, number of signal averages $[\mathrm{NSA}]=2$ ) to measure renal artery flow at 20 timepoints over the cardiac cycle. Scans were acquired for both the left and right renal arteries.

$\mathrm{T}_{2}{ }^{*}$-weighted images were acquired with a multiple gradient-recalled echo image sequence $(\mathrm{TR}=109 \mathrm{~ms}$, $\left.\operatorname{tip}=30^{\circ}, \mathrm{NSA}=1\right)$. Contrast-enhanced $3 \mathrm{D}$ datasets showing early- and late-stage contrast enhancement were acquired using a 3D gradient echo imaging sequence $(\mathrm{TR}=$ $5.1 \mathrm{~ms}, \mathrm{TE}=1.56 \mathrm{~ms}$, flip $\left.=30^{\circ}, \mathrm{NSA}=1\right)$. Contrast agent (Magnevist, Bayer HealthCare, Morristown, NJ, USA) was administered intravenously at a dose of $0.2 \mathrm{ml} / \mathrm{kg}$ body weight, using an automated injector system (MedRad Spectris Solaris EP, Warrendale, PA, USA) at a flow rate of $2.0 \mathrm{ml} / \mathrm{s}$, followed by a $20 \mathrm{ml}$ saline flush. $3 \mathrm{D}$ image datasets were acquired at $30 \mathrm{~s}$ and $5 \mathrm{~min}$ after contrast agent administration.

Data analysis Data from kidneys with more than one renal artery were rejected. Phase contrast angiography data were analysed with ViewForum software's 'Cardiac Analysis' tool (Philips Medical Systems). A region of interest (ROI) was drawn around the renal artery on a magnitude phase contrast image, and this ROI propagated to the calculated flow maps. Flow through the renal artery per cardiac stroke was then calculated from these data, and corrected for body surface area.

$R_{2}{ }^{*}$ maps were generated from multiecho gradient echo images using in-house software written in Matlab (The Mathworks, Natick, MA, USA). The change in cortical and medullary $R_{2}{ }^{*}$ following water loading, and heterogeneity in $R_{2}{ }^{*}$, was determined from cortical and medullary ROIs that were chosen based on anatomic information from the $\mathrm{T}_{1}$-weighted $\mathrm{S}_{0}$ maps and from $3 \mathrm{D}$ contrast-enhanced image datasets.

Renal cortical and medullary volumes were determined from contrast-enhanced 3D gradient echo images. Baseline images acquired before contrast agent administration were 
Table 1 Clinical characteristics of the study volunteers

\begin{tabular}{|c|c|c|c|c|}
\hline \multirow[t]{2}{*}{ Characteristic } & \multirow[t]{2}{*}{ Type 1 normoalbuminuric } & \multirow[t]{2}{*}{ Type 1 microalbuminuric } & \multicolumn{2}{|c|}{ Type 1 microalbuminuric subgroup } \\
\hline & & & On RAAS & Off RAAS \\
\hline M:F & $4: 5$ & $4: 4$ & $3: 3$ & $3: 3$ \\
\hline Age (years) & $40.1(31-50)$ & $42.5(33-52)$ & $41.5(33-52)$ & $41.5(33-52)$ \\
\hline Duration (years) & $28.4(21-38)$ & $27.0(22-37)$ & $28.2(21-37)$ & $28.2(21-37)$ \\
\hline Retinopathy (N:B:P) & $3: 6: 0$ & $0: 2: 6$ & $0: 1: 5$ & $0: 1: 5$ \\
\hline Systolic blood pressure $(\mathrm{mmHg})$ & $123 \pm 9$ & $137 \pm 13^{\mathrm{a}}$ & $136 \pm 14$ & $140 \pm 9$ \\
\hline Diastolic blood pressure $(\mathrm{mmHg})$ & $70 \pm 7$ & $86 \pm 12^{b}$ & $85 \pm 14$ & $85 \pm 5$ \\
\hline $\mathrm{HbA}_{1 \mathrm{c}}(\%)$ & $8.0 \pm 1.1$ & $9.3 \pm 1.6$ & $8.8 \pm 1.5$ & $8.8 \pm 1.8$ \\
\hline Serum creatinine $(\mu \mathrm{mol} / \mathrm{l})$ & $86 \pm 8$ & $99 \pm 10^{\mathrm{a}}$ & $96 \pm 10$ & $88 \pm 9$ \\
\hline eGFR $\left(\mathrm{ml} \mathrm{min}-1.73 \mathrm{~m}^{-2}\right)$ & $83.4 \pm 10.6$ & $71.6 \pm 13.7$ & $74.1 \pm 13.8$ & $82.5 \pm 16.1$ \\
\hline $\operatorname{ACR}(\mathrm{mg} / \mathrm{mmol})$ & $0.10(0.10-0.5)$ & $21.6(5.5-104.5)$ & $42.2(5.5-104.5)$ & $55.3(11.0-81.7)$ \\
\hline Total cholesterol (mmol/l) & $4.4 \pm 0.6$ & $4.8 \pm 0.9$ & $4.7 \pm 0.6$ & ND \\
\hline
\end{tabular}

F, female; M, male; N:B:P, none: background: proliferative; ND, no data

${ }^{\mathrm{a}} p<0.05$ T1micro vs T1normo

${ }^{\mathrm{b}} p<0.01$ T1micro vs T1normo

subtracted from images acquired $30 \mathrm{~s}$ (early cortical enhancement) and $5 \mathrm{~min}$ (late cortical and medullary enhancement) after contrast agent administration. Data were analysed using OsiriX software version 3.5.1 (www.osirix-viewer.com).

Where appropriate, data were standardised by scaling to body surface area [24]. Mean arterial pressure was calculated as one-third systolic plus two-thirds diastolic pressure. Renal vascular resistance was calculated as mean arterial pressure $(\mathrm{mmHg})$ divided by total renal blood flow (sum of flow through right plus left renal arteries; $\mathrm{ml} \mathrm{min}^{-1} 1.73 \mathrm{~m}^{-2}$ ) [8].

Detailed magnetic resonance imaging and data analysis methodology is provided in the Electronic supplementary material (ESM).

Statistical analysis Statistical analysis was performed using SPSS 15.0 (IBM, Chicago, IL, USA). Data are presented as mean \pm SD or median (range). Statistical analysis of datasets employed a one-way ANOVA with Scheffe post hoc multiple comparisons. Correlations were by Pearson analysis.

\section{Results}

Volumetric data The contrast-enhanced imaging allowed clear definition of cortex and medulla. Figure 1 shows representative difference images generated by subtracting baseline images from early- and late-stage contrastenhanced images acquired at $30 \mathrm{~s}$ and $5 \mathrm{~min}$ after administration of contrast agent. Typical ROIs describing enhancement volumes are shown in green. Mean cortical and medullary volumes were very similar in control, T1Normo and T1Micro participants, and the ratio of cortex to medulla was similar in all three groups (Table 2).

Phase contrast angiography Data from a representative control participant phase contrast angiography dataset are shown in Fig. 2. The location and orientation of the angiography scan relative to the right renal artery is shown (Fig. 2a), as is a magnitude image from the angiography dataset with a region of interest encompassing the renal artery (Fig. 2b). Figure 2c shows a plot of mean renal artery flow at 20 timepoints over the cardiac cycle, acquired before water loading.

Before water loading, renal artery flow was $740 \pm 205$ in the control participants and $591 \pm 128 \mathrm{ml} \mathrm{min}^{-1} 1.73 \mathrm{~m}^{-2}$ in
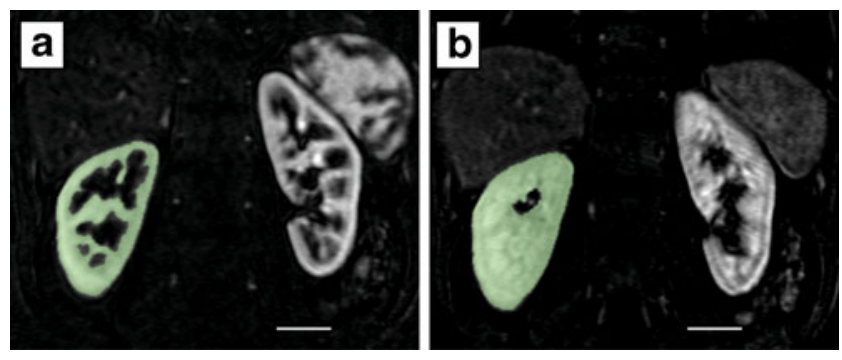

Fig. 1 Representative difference images generated by subtracting baseline images from contrast-enhanced images acquired at $30 \mathrm{~s} \mathrm{(a)}$ and 5 min (b) after administration of contrast agent. The images show early (a) and late-stage (b) enhancement due to perfusion with blood containing this agent. Example regions of interest used to calculate cortical (a) and cortical+medullary (b) volumes are shown in green. Scale bars, $3 \mathrm{~cm}$ 
the whole diabetes cohort $(p=0.035)$. There was no difference between the normoalbuminuric and microalbuminuric diabetic groups $\left(583 \pm 135\right.$ vs $603 \pm 128 \mathrm{ml} \mathrm{min}^{-1}$ $1.73 \mathrm{~m}^{-2}$; Table 2). Although flow in the two diabetic groups was approximately $20 \%$ lower than in the control group, the differences between individual groups did not reach statistical significance (ANOVA $p=0.110$ ). After water loading, when all participants were analysed together, renal artery flow increased significantly (pre-water load $650 \pm 176$ vs post $713 \pm 186 \mathrm{ml} \mathrm{min}^{-1} 1.73 \mathrm{~m}^{-2}$; paired Student's $t$ test $p=0.003)$. Post-water load flow was significantly lower in the whole diabetes cohort (control $812 \pm 229$ vs diabetic $654 \pm 115 \mathrm{ml} \mathrm{min}^{-1} 1.73 \mathrm{~m}^{-2}$; $p=0.028$ ).

However, in the three individual groups, the change in renal artery flow with water loading was not significant (Table 2). Withdrawal of RAAS inhibition did not bring about any change in renal artery flow in the microalbuminuric patients (On 620 136 vs Off $581 \pm$ $181 \mathrm{ml} \mathrm{min}^{-1} 1.73 \mathrm{~m}^{-2}$ ).

Mean arterial pressure was $17 \%$ higher in the microalbuminuric group than in the normoalbuminuric and control participants $(p<0.01$; Table 2). RVR increased stepwise from control to normoalbuminuric to microalbuminuric (ANOVA $p=0.012$ ), with significant individual differences between control and microalbuminuric participants $(p=0.011)$. RVR correlated inversely with eGFR in the normoalbuminuric volunteers $(r=-0.905, p=0.001)$ but not in the microalbuminuric patients on $(r=-0.528$, $p=0.361)$ or off $(r=-0.203, p=0.700)$ RAAS inhibition (Fig. 3).

$T_{2}{ }^{*}$ Measurements Figure 4a shows a representative kidney $R_{2}{ }^{*}$ map from a control participant. Heterogeneity in transverse relaxivity is seen across the kidney. A $\mathrm{T}_{1}$-weighted image from the same participant is shown in Fig. 4b, which was acquired at the same resolution, field of view and orientation as the $R_{2}{ }^{*}$ map, showing contrast between cortex and medulla. Figure 4c shows an earlyenhancement $T_{1}$-weighted image with approximately the same image orientation as the $R_{2}{ }^{*}$ map, acquired $30 \mathrm{~s}$ after administration of contrast agent. Clear delineation of cortex and medulla can be seen on the contrast-enhanced image.

Masks of cortical and medullary regions, drawn by hand on the $\mathrm{T}_{1}$-weighted image and informed by the contrastenhanced image were superimposed on the $R_{2}{ }^{*}$ map to form Fig. 4 d, e. These masked images allow separate assessment of $R_{2}{ }^{*}$ heterogeneity within the two tissue types. Considerable $R_{2}{ }^{*}$ heterogeneity is observed within the medulla regions of the kidney $(28 \pm 9 \mathrm{~ms})$. Mean cortical and medullary $R_{2}{ }^{*}$ measurements calculated from these regions of interest in a subset of five randomly selected members of the control group, before and after water loading, are shown in Fig. 5. The large error bars in the medulla data reflect the heterogeneous $R_{2}{ }^{*}$ of these regions, and no statistically significant change in cortical or medullary $R_{2}{ }^{*}$ was observed on water loading. As the data from the volunteer group were not capable of demonstrating a small change in medullary $R_{2}{ }^{*}$ on water loading (as reported in studies of volunteers with type 2 diabetes), no further analysis of $R_{2}{ }^{*}$ data was performed.

\section{Discussion}

Volumetric data Contrast-enhanced imaging provided good discrimination between cortex and medulla and demonstrated no difference in cortical or medullary volumes between the three groups. Total kidney volume is normal or even slightly increased in short-duration type 1 diabetic patients when assessed by renal ultrasound, and is influenced by blood glucose [25, 26]. Here, using MRI, we demonstrate normal cortical and medullary volumes, with normal cortical:medullary ratios, in our type 1 diabetic

Table 2 Study volunteer morphological and haemodynamic properties

\begin{tabular}{lccc}
\hline Property & Control & Type 1 normoalbuminuric & Type 1 microalbuminuric \\
\hline Cortical volume $\left(\mathrm{cm}^{3} / 1.73 \mathrm{~m}^{2}\right)$ & $108 \pm 16$ & $112 \pm 21$ & $111 \pm 10$ \\
Medullary volume $\left(\mathrm{cm}^{3} / 1.73 \mathrm{~m}^{2}\right)$ & $35 \pm 14$ & $29 \pm 10$ & $33 \pm 6$ \\
Cortical/medullary ratio & $3.5 \pm 1.4$ & $4.2 \pm 1.7$ & $3.4 \pm 0.7$ \\
Pre-water load renal artery flux $\left(\mathrm{ml} \mathrm{min}^{-1} 1.73 \mathrm{~m}^{-2}\right)$ & $740 \pm 205$ & $583 \pm 135$ & $603 \pm 128$ \\
Post-water load renal artery flux $\left(\mathrm{ml} \mathrm{min}^{-1} 1.73 \mathrm{~m}^{-2}\right)$ & $812 \pm 229$ & $638 \pm 109$ & $673 \pm 129$ \\
Mean arterial pressure $(\mathrm{mmHg})$ & $87 \pm 10$ & $88 \pm 6$ & $103 \pm 11^{\mathrm{a}}$ \\
Renal vascular resistance $\left(\mathrm{mmHg} \mathrm{ml} \mathrm{min}^{-1} 1.73 \mathrm{~m}^{-2}\right)$ & $0.061 \pm 0.018$ & $0.077 \pm 0.014$ & $0.093 \pm 0.024^{\mathrm{b}}$ \\
\hline
\end{tabular}

${ }^{\mathrm{a}} p<0.01$ vs control and type 1 normoalbuminuric diabetic participants

${ }^{\mathrm{b}} p<0.05$ vs control participants 

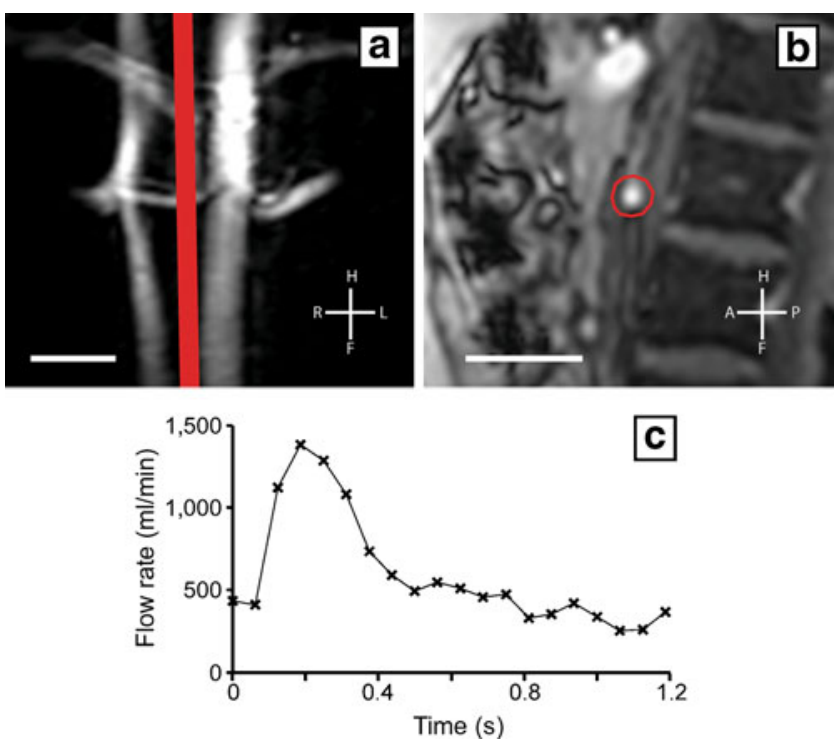

Fig. 2 Data from a representative phase contrast angiography dataset from a control participant. The location and orientation of the angiography scan relative to the right renal artery is shown (a), as is a magnitude image from the angiography dataset with a region of interest encompassing the renal artery (red circle) (b). c A representative plot of mean renal artery flow at 20 timepoints over the cardiac cycle. Scale bar, $3 \mathrm{~cm}$

patients. The normoalbuminuric patients were of long duration, in stable glucose control, with persistently normal albumin excretion. Kidney volume was normal. Cortical and medullary volumes in the microalbuminuric patients were also similar, in spite of likely underlying marked glomerular and medullary structural changes. This finding is supported by previous observations of normal total renal volume in advanced diabetic kidney disease, in contrast to reduced volume in other chronic kidney diseases [3].

Renal haemodynamics We have demonstrated a stepwise increase in RVR from control to normoalbuminuric to microalbuminuric participant, using non-invasive MRI methods, and controlling for age, blood glucose and use
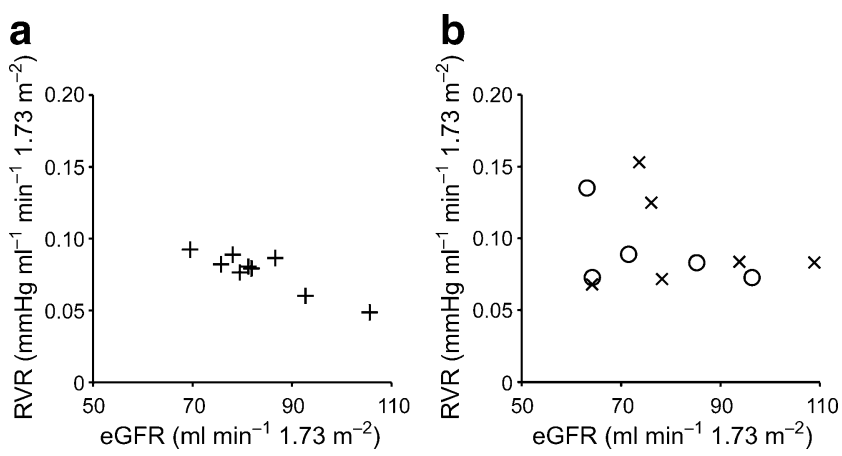

Fig. 3 Relationship between eGFR and renal vascular resistance in normoalbuminuric type 1 diabetic patients (a) and microalbuminuric patients (b) on (circles) and off (crosses) inhibitors of the RAAS of RAAS inhibitors. A significant decrease in renal artery flow was observed in the combined type 1 diabetic volunteers compared with control volunteers, although individual group differences did not reach statistical significance. Previous studies of renal haemodynamics in diabetes have typically reported renal plasma flow rather than renal blood flow, measured by clearance studies using radioisotopes or other tracers, and a good correlation between MR and tracer studies has been reported [27]. The MRI-derived values for renal blood flow in our control participants are of the same order of magnitude as previously reported using MRI [27] and clearance methods [8]. Clearance studies have suggested increased [28] or normal $[26,29]$ effective renal plasma flow in younger normoalbuminuric type 1 patients with relatively short disease duration. However, our normoalbuminuric patients were older, with much longer durations of diabetes. Several studies have demonstrated similar effective renal plasma flow in normoalbuminuric and microalbuminuric type 1 diabetic patients $[30,31]$.

The pattern of flow through the renal arteries was similar in our diabetic and control groups, suggesting that the reduction in flow was not due to haemodynamically significant narrowing of the renal artery, but attributable to increased intrarenal vascular resistance. RVR, calculated from clearance of para-aminohippurate, is reduced in young type 1 diabetic patients without complications, and increases with age, independent of diabetes duration [8]. In contrast, in control participants, there is no or a very weak effect of age on RVR [8]. Increasing systemic arterial stiffness accompanies the increase in RVR in diabetes [8], suggesting that in our older, long duration normoalbuminuric diabetic patients, RVR may be secondary to stiffening in the intralobular arteries and arterioles.

RVR was also increased in our microalbuminuric type 1 diabetic patients. However, in contrast to the inverse correlation between eGFR and RVR in our normoalbuminuric diabetic volunteers, there was no significant relationship between eGFR and RVR in the microalbuminuric patients, on or off RAAS inhibition. This suggests that additional factor(s), perhaps including loss of autoregulation and raised intraglomerular pressure, contribute to the increased RVR in microalbuminuric patients.

Our data represent a global whole kidney perfusion measurement. However, MRI techniques can provide regional renal haemodynamic information. Dynamic contrast-enhanced MRI can report on perfusion and glomerular filtration [32] and contrast agent-free methods are being developed to allow perfusion methods without the risks of NSF [33]. Such approaches offer significant advantages in detecting pathological changes that may be regional or heterogeneous in their effects, and are forming the basis of our future studies. 

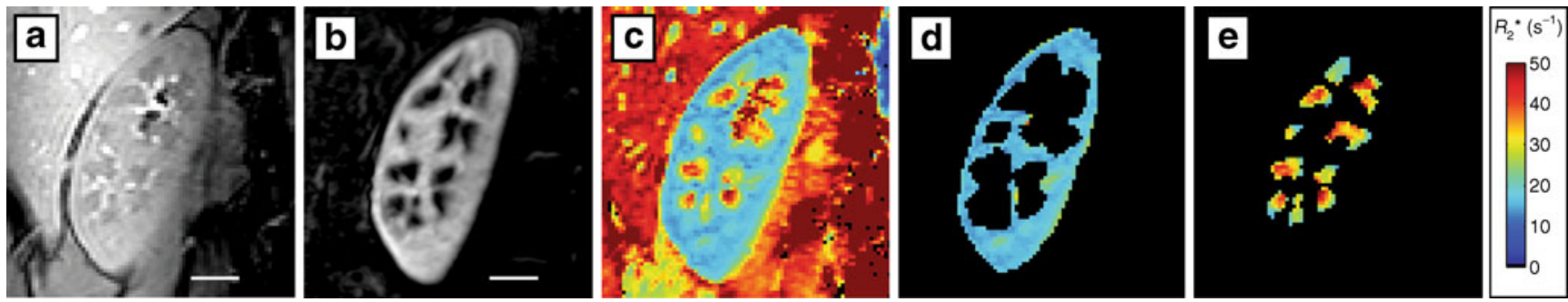

Fig. 4 a A representative kidney $R_{2}{ }^{*}$ map from a control participant, demonstrating heterogeneity in transverse relaxivity across the kidney. b $A T_{1}$-weighted image from the same participant, acquired at the same resolution, field of view and orientation as the $R_{2}{ }^{*}$ map. Contrast between cortex and medulla is observed in this image. c An earlyenhancement $\mathrm{T}_{1}$-weighted image with approximately the same image orientation as the $R_{2}{ }^{*}$ map, acquired $30 \mathrm{~s}$ after administration of contrast agent. Clear delineation of cortex and medulla can be seen on the contrast-enhanced image. $\mathbf{d}$ and $\mathbf{e}$ Masks of cortical and medullary regions, drawn by hand from the $\mathrm{T}_{1}$-weighted image with guidance from the contrast-enhanced image and superimposed on the $R_{2}{ }^{*}$ map, to allow separate assessment of $R_{2}{ }^{*}$ heterogeneity within the two tissue types. Scale bars, $2 \mathrm{~cm}$

induce bias into results as the region of interest is chosen on maps of the parameter to be measured. We noticed considerable $R_{2}^{*}$ heterogeneity across the kidney, particularly within the medulla, as reported in other kidney BOLD studies [37]. Faced with this observation, it became difficult to employ a robust and rigorous approach to selecting ROI position on $T_{2}{ }^{*}$ or $R_{2}{ }^{*}$ maps without bias towards a subgroup of medullary $R_{2}{ }^{*}$ values.

We used $\mathrm{T}_{1}$-weighted and contrast agent-enhanced images to identify the medulla. Medullary $R_{2}{ }^{*}$ heterogeneity was observed over $3-6 \mathrm{~mm}$, which is of the same order as the slice thickness employed for $R_{2}{ }^{*}$ map data acquisition $(5 \mathrm{~mm})$. Thus, an inaccuracy in slice repositioning of just 1-3 $\mathrm{mm}$ has the potential to cause a large change in the measured $R_{2}{ }^{*}$ from a small medullary ROI. $R_{2}{ }^{*}$ map data acquisition occurred during breath-holding, and participants were repositioned in the scanner between the two $R_{2}{ }^{*}$ map scans. Thus, image slice positioning was not identical for the pre- and post-water loading scan sessions. Our data demonstrate that the placement of small ROIs by hand on $R_{2}{ }^{*}$ maps, for comparison of datasets that may have minor differences in slice positioning, may be subject to repositioning errors and analysis bias. However, an alternative approach intended to be more robust (selection of ROIs to cover the $R_{2}{ }^{*}$ map areas that could be unambiguously assigned to medulla) demonstrated a heterogeneity of $R_{2}{ }^{*}$ sufficient to mask any small changes. Thus, our data suggest that studies of medullary $R_{2}{ }^{*}$ require either an unbiased method for placement of small ROIs, or a change in $R_{2}{ }^{*}$ that is sufficiently large to allow detection in an ROI encompassing the entire medulla region. Thus, we are unable to confidently present changes in medullary $R_{2}{ }^{*}$ in response to water loading, nor to assign their origin to BOLD effects. Our data demonstrated no statistically significant changes in cortical or medullary $R_{2}{ }^{*}$ on water loading in the control participants studied.

The strengths of our study include the detailed phenotyping of our patients and the state of the art MRI
Fig. $5 R_{2}{ }^{*}$ measurements from cortical (a) and medullary (b) regions of interest from five control volunteers. Dark grey bars, pre-water loading; light grey bars, post-water loading 
methodology. Group size was relatively small, and may be why some observed differences between the two groups of diabetic volunteers did not reach statistical significance. Because of the complex nature of the study, we used eGFR rather than a direct measure. However, eGFR calculated by the CKD-EPI equation is much more accurate than the MDRD equation, particularly in individuals with eGFR $>60 \mathrm{ml} \mathrm{min}^{-1} 1.73 \mathrm{~m}^{-2}$, as in our study [38].

Conclusions We have used a range of magnetic resonance imaging techniques to identify pathophysiological changes associated with early diabetic kidney disease. We have demonstrated normal cortical and medullary volumes in type 1 diabetic patients with and without microalbuminuria. Renal artery flow was $20 \%$ lower in diabetic compared with control participants, with no difference in flow between microalbuminuric and normoalbuminuric patients. Renal vascular resistance was reduced in normoalbuminuric and microalbuminuric type 1 diabetic patients compared with control participants, and we postulate different mechanisms between these two volunteer groups resulting in these differences. We demonstrated considerable heterogeneity in medullary $R_{2}{ }^{*}$, which compromised attempts to measure subtle changes in $R_{2}{ }^{*}$ that have been ascribed to tissue oxygenation. Our data suggest that a robust method of selecting medullary ROIs on $R_{2}{ }^{*}$ maps may be required for kidney BOLD imaging.

Acknowledgements We thank the study volunteers for their participation. Thanks to our radiographers (L. Morris, C. Smith and T. Hodgson) and to M. Clemence and M. Pike (Philips Medical Systems) for technical assistance. M. J. Chen provided expert nursing assistance. This study was funded by the Diabetes Research and Wellness Foundation.

Duality of interest statement The authors declare that there is no duality of interest associated with this manuscript.

\section{References}

1. Byrne C, Ford D, Gilg J, Ansell D, Feehally J (2009) UK Renal Registry 12th Annual Report: Chapter 3: UK ESRD incident rates in 2008: national and centre-specific analyses. Nephron Clin Pract 115(Suppl 1):c9-39

2. Marshall SM, Flyvbjerg A (2006) Prevention and early detection of vascular complications of diabetes. BMJ 333:475-480

3. Ellis EN, Steffes MW, Goetz FC, Sutherland DE, Mauer SM (1985) Relationship of renal size to nephropathy in type 1 (insulin-dependent) diabetes. Diabetologia 28:12-15

4. Christiansen T, Rasch R, Stodkilde-Jorgensen H, Flyvbjerg A (1997) Relationship between MRI and morphometric kidney measurements in diabetic and non-diabetic rats. Kidney Int 51:50-56

5. Brenner BM, Hostetter TH, Olson JL, Rennke HG, Venkatachalam MA (1981) The role of glomerular hyperfiltration in the initiation and progression of diabetic nephropathy. Acta Endocrinol Suppl (Copenh) 242:7-10
6. Mogensen CE, Andersen MJ (1975) Increased kidney size and glomerular filtration rate in untreated juvenile diabetes: normalization by insulin-treatment. Diabetologia 11:221-224

7. Ditzel J, Junker K (1972) Abnormal glomerular filtration rate, renal plasma flow, and renal protein excretion in recent and short-term diabetics. BMJ 2:13-19

8. Cherney DZ, Reich HN, Miller JA et al (2010) Age is a determinant of acute hemodynamic responses to hyperglycemia and angiotensin II in humans with uncomplicated type 1 diabetes mellitus. Am J Physiol Regul Integr Comp Physiol 299:R206-214

9. Miyata T, de Strihou CY (2010) Diabetic nephropathy: a disorder of oxygen metabolism? Nat Rev Nephrol 6:83-95

10. Brezis M, Rosen S (1995) Hypoxia of the renal medulla-its implications for disease. N Engl J Med 332:647-655

11. Grenier N, Hauger O, Cimpean A, Perot V (2006) Update of renal imaging. Semin Nucl Med 36:3-15

12. Cheong B, Muthupillai R, Rubin MF, Flamm SD (2007) Normal values for renal length and volume as measured by magnetic resonance imaging. Clin J Am Soc Nephrol 2:38-45

13. Ogawa S, Lee TM, Kay AR, Tank DW (1990) Brain magnetic resonance imaging with contrast dependent on blood oxygenation. Proc Natl Acad Sci U S A 87:9868-9872

14. Prasad PV, Chen Q, Goldfarb JW, Epstein FH, Edelman RR (1997) Breath-hold R2 ${ }^{*}$ mapping with a multiple gradient-recalled echo sequence: application to the evaluation of intrarenal oxygenation. J Magn Reson Imaging 7:1163-1165

15. Prasad PV, Edelman RR, Epstein FH (1996) Noninvasive evaluation of intrarenal oxygenation with BOLD MRI. Circulation 94:3271-3275

16. Prasad PV, Epstein FH (1999) Changes in renal medullary $\mathrm{pO}_{2}$ during water diuresis as evaluated by blood oxygenation leveldependent magnetic resonance imaging: effects of aging and cyclooxygenase inhibition. Kidney Int 55:294-298

17. Tumkur SM, Vu AT, Li LP, Pierchala L, Prasad PV (2006) Evaluation of intra-renal oxygenation during water diuresis: a time-resolved study using BOLD MRI. Kidney Int 70:139-143

18. Mason RP (2006) Non-invasive assessment of kidney oxygenation: a role for BOLD MRI. Kidney Int 70:10-11

19. Hofmann L, Simon-Zoula S, Nowak A et al (2006) BOLD-MRI for the assessment of renal oxygenation in humans: acute effect of nephrotoxic xenobiotics. Kidney Int 70:144-150

20. Economides PA, Caselli A, Zuo CS et al (2004) Kidney oxygenation during water diuresis and endothelial function in patients with type 2 diabetes and subjects at risk to develop diabetes. Metabolism 53:222-227

21. Epstein FH, Veves A, Prasad PV (2002) Effect of diabetes on renal medullary oxygenation during water diuresis. Diabetes Care 25:575-578

22. Levey AS, Stevens LA, Schmid CH et al (2009) A new equation to estimate glomerular filtration rate. Ann Intern Med 150:604612

23. Christiansen JS, Frandsen M, Parving HH (1981) Effect of intravenous glucose infusion on renal function in normal man and in insulin-dependent diabetics. Diabetologia 21:368-373

24. Haycock GB, Schwartz GJ, Wisotsky DH (1978) Geometric method for measuring body surface area: a height-weight formula validated in infants, children, and adults. J Pediatr 93:62-66

25. Christiansen JS, Gammelgaard J, Tronier B, Svendsen PA, Parving HH (1982) Kidney function and size in diabetics before and during initial insulin treatment. Kidney Int 21:683-688

26. Tuttle KR, Bruton JL, Perusek MC, Lancaster JL, Kopp DT, DeFronzo RA (1991) Effect of strict glycemic control on renal hemodynamic response to amino acids and renal enlargement in insulin-dependent diabetes mellitus. $\mathrm{N}$ Engl J Med 324:1626-1632 
27. Wolf RL, King BF, Torres VE, Wilson DM, Ehman RL (1993) Measurement of normal renal artery blood flow: cine phasecontrast MR imaging vs clearance of $p$-aminohippurate. AJR Am J Roentgenol 161:995-1002

28. Christensen CK, Christiansen JS, Christensen T, Hermansen K, Mogensen CE (1986) The effect of six months continuous subcutaneous insulin infusion on kidney function and size in insulin-dependent diabetics. Diabet Med 3:29-32

29. Luik PT, Hoogenberg K, van Der Kleij FG et al (2002) Short-term moderate sodium restriction induces relative hyperfiltration in normotensive normoalbuminuric type I diabetes mellitus. Diabetologia 45:535-541

30. Trevisan R, Bruttomesso D, Vedovato M et al (1998) Enhanced responsiveness of blood pressure to sodium intake and to angiotensin II is associated with insulin resistance in IDDM patients with microalbuminuria. Diabetes 47:1347-1353

31. Poulsen PL, Juhl B, Ebbehoj E, Klein F, Christiansen C, Mogensen CE (1997) Elevated ambulatory blood pressure in microalbuminuric IDDM patients is inversely associated with renal plasma flow. A compensatory mechanism? Diabetes Care 20:429-432

32. Artunc F, Yildiz S, Boss A et al. (2011) Measurement of glomerular filtration rate using dynamic magnetic resonance imaging in patients with chronic kidney disease. J Nephrol. doi: $10.5301 / \mathrm{JN} .2010 .5978$
33. Martirosian P, Boss A, Schraml C et al (2010) Magnetic resonance perfusion imaging without contrast media. Eur J Nucl Med Mol Imaging 37:S52-64

34. Li LP, Storey P, Pierchala L, Li W, Polzin J, Prasad P (2004) Evaluation of the reproducibility of intrarenal $\mathrm{R} 2 *$ and DeltaR2* measurements following administration of furosemide and during waterload. J Magn Reson Imaging 19:610-616

35. Simon-Zoula SC, Hofmann L, Giger A et al (2006) Non-invasive monitoring of renal oxygenation using BOLD-MRI: a reproducibility study. NMR Biomed 19:84-89

36. Li LP, Ji L, Santos EA, Dunkle E, Pierchala L, Prasad P (2009) Effect of nitric oxide synthase inhibition on intrarenal oxygenation as evaluated by blood oxygenation leveldependent magnetic resonance imaging. Invest Radiol 44: $67-73$

37. Zuo CS, Rofsky NM, Mahallati H et al (2003) Visualization and quantification of renal R2* changes during water diuresis. J Magn Reson Imaging 17:676-682

38. Stevens LA, Schmid CH, Greene T et al (2010) Comparative performance of the CKD Epidemiology Collaboration (CKD-EPI) and the Modification of Diet in Renal Disease (MDRD) Study equations for estimating GFR levels above $60 \mathrm{~mL} / \mathrm{min} / 1.73 \mathrm{~m}^{2}$. Am J Kidney Dis 56:486-495 